\title{
Ensayos exploratorios en macetas sobre fertilidad de suelos del Chaco Oriental Argentino
}

\author{
C.E.Tomei, P. Regonat, C.E. Tomei(h), M.E. Castelan y G. Arce \\ Instituto Agrotécnico "Pedro M. Fuentes Godo", UNNE, \\ Las Heras 727, (3500) Resistencia, Chaco, Argentina.
}

\section{Resumen.}

Se estudia el efecto del agregado de $\mathrm{P}, \mathrm{K}, \mathrm{Ca}, \mathrm{Mg}$ y $S$ en cuatro suelos del Chaco Oriental, República Argentina y de $\mathrm{Mo}, \mathrm{Cu}, \mathrm{Zn}$ y $\mathrm{B}$ en dos de ellos. Se aplicó la técnica del cultivo intensivo en macetas, en invernadero, la planta utilizada fue Trifolium repens cv. Haifa. El diseño estadístico fue completamente al azar con tres repeticiones. Se realizó el análisis de la variancia, las diferencias entre medias de los tratamientos se probaron con el test de Tukey, nivel $5 \%$. Los resultados indican que los suelos estudiados pueden presentar restricciones de nutrientes minerales que limitan el establecimiento y persistencia de leguminosas forrajeras invernales. Uno de los suelos estudiados, Los Laureles (Provincia de Santa Fe), tiene como deficiencias primarias $\mathrm{P}$ y $\mathrm{Zn}$, siendo el Mg y S deficiencias secundarias. En dos suelos de la Provincia del Chaco hay deficiencias de $\mathrm{Ca}$ y $\mathrm{Mg}$. Solo uno de los suelos (Provincia de Formosa) no presenta deficiencias minerales en los nutrientes analizados.

Palabras clave: Ensayos en macetas, leguminosas forrajeras, deficiencias nutritivas, Trifolium repens.

Titulo abreviado: Ensayos exploratorios en macetas...

\section{Summary}

The effect of $P, K \mathrm{Ca}, \mathrm{Mg}$ and $\mathrm{S}$ addition on four soils from Eastern Chaco area (Argentine) was studied and $\mathrm{Mo}, \mathrm{Cu}, \mathrm{Zn}$ and $\mathrm{B}$ were added in two of them. A greenhouse trial pot applying the intensive culture concept was done. A completely randomized desing with three replications was used. Differences were tested using Tukey, at 5 $\%$ level. Results showed that soils from Eastern Chaco can display mineral nutrients restriction that reduce winter forage legumes establishment and persistence. $\mathrm{P}$ and $\mathrm{Zn}$ as primary deficiency and $\mathrm{Mg}$ and $\mathrm{S}$ as secondary deficiency were present in one soil from Santa Fe Province (Los Laureles soil). $\mathrm{Ca}$ and $\mathrm{Mg}$ deficiency were present in both soils from Chaco Province. Mineral deficiencies were not detected of the soil from Formosa Province.

Key words: Pots trials, forrage legumes, nutrient deficiency, Trifolium repens.

\section{Introducción}

De los cultivos forrajeros la alfalfa (Medicago sativa) y el melilotus (Melilotus alba var. annua) son los más difundidos en la Región Chaqueña Argentina, sin embargo estos se han concentrado en el centro y el oeste de la misma, sobre todo en la Provincia del Chaco. En la franja delimitada por los ríos Paraná y Paraguay al este y el meridiano de $60^{\circ} \mathrm{O}$, los intentos de implantación de leguminosas forrajeras como el melilotus, la alfalfa o el trébol blanco (Trifolium repens) han fracasado con mucha frecuencia. Esto sucede tanto en campos del norte de la Provincia de Santa Fe donde se realiza la fertilización fosforada en la siembra de pasturas que incluyen leguminosas, como en el Este de la Provincia del Chaco. Se supone que algunas de las causas de los fracasos en la implantación de leguminosas forrajeras de invierno en el Chaco Oriental Argentino provienen de restricciones en la disponibilidad de algunos nutrientes minerales.

Para el estudio de deficiencias minerales en suelos de Corrientes, Tomei et al, (1974a y 1995b) han utilizado el cultivo de leguminosas forrajeras en macetas. Estos trabajos mostraron la existencia de deficiencias en $\mathrm{P}$, Ca y algunas veces $\mathrm{K}$ para el $T$. repens, Lotus corniculatus y $M$. hispida en esos suelos. Estos autores siguieron el concepto de cultivo intensivo propuesto por Chaminade et al (1965). El método permite hacer un diagnóstico preliminar de las deficiencias y precisar los nutrientes que serán objeto de ensayos de campo (Schenckel et al, 1971). Aunque es un test $\sin$ 
valor absoluto por si mismo (Chaminade,1968), tiene el mérito de indicar cuáles son las deficiencias de nutrientes minerales y su gravedad (Roche y Velly, 1965). Los diagnósticos de carencias en suelos tropicales con el uso de macetas, son coincidentes con los resultados de campo para $\mathrm{P}, \mathrm{K}, \mathrm{Ca}$ y Mg (Roche, 1967).

El objetivo de esta contribución fue probar la hipótesis de la existencia de restricciones edáficas nutritivas minerales para el Trifolium repens $\mathrm{cv}$. Haifa, en suelos del Chaco Oriental Argentino.

\section{Materiales y Métodos.}

Las experiencias se ejecutaron en el invernáculo de la Facultad de Ciencias Agrarias, Universidad Nacional del Nordeste, Corrientes, Argentina. Se estudiaron dos suelos de la Provincia del Chaco, uno de la Provincia de Formosa y otro del norte de la Provincia de Santa Fe. La ubicación geográfica de los sitios donde se extrajeron muestras compuestas de los primeros $20 \mathrm{~cm}$ de profundidad son los siguientes:

Colonia Popular: Sitio ubicado a $20 \mathrm{~km}$ al Noroeste de la ciudad de Resistencia (27을 $30^{\prime} \mathrm{S}$; $58^{\circ} 54^{\prime}$ W), Provincia del Chaco. Ambiente de pastizal dominado por Elionorus muticus, relieve normal, suelo clasificado como argiudol típico
(Ledesma, 1990).

PuertoTirol: El sitio de extracción se encuentra a $15 \mathrm{~km}$ al Oeste de la ciudad de Resistencia (27은 $\left.30^{\prime} \mathrm{S} ; 58^{\circ} 54^{\prime} \mathrm{W}\right)$, Provincia del Chaco. Ambiente de pastizal de composición botánica heterogénea dominado por Eliunorus muticus y Sporobolus poiretii. El suelo está clasificado como argiudol típico (Ledesma, 1990).

A Mbiguá: Sitio ubicado encuentra a $40 \mathrm{~km}$ al Sur de la ciudad de Formosa $\left(26^{\circ} 43^{\prime} 18^{\prime \prime} \mathrm{S} ; 58^{\circ}\right.$ $50^{\prime} \mathrm{W}$ ), loma tendida a $2 \mathrm{~km}$ del Arroyo Mbiguá. Suelo clasificado como Haplustol óxico (Ledesma, 1990). En los dos años previos al muestreo se cultivó sorgo forrajero.

Los Laureles: El sitio está ubicado en Los Laureles $\left(29^{\circ} 22^{\prime} 23^{\prime \prime} \mathrm{S} ; 59^{\circ} 41^{\prime} 25^{\prime \prime} \mathrm{W}\right)$ a $24 \mathrm{~km}$ al Sur de la ciudad de Reconquista, Provincia de Santa Fe. Es un suelo con 30 años de cultivo de algodón, maíz y soja, sin fertilización. El suelo es un argiudol vértico (Espino y Seveso, 1983), horizonte $A 1$ de textura franco arcillo limosa, B2 textural, arcilloso. De permeabilidad moderada a moderadamente lenta debido a la presencia del B2t.

La clasificación edafológica y las propiedades físico - químicas de los suelos estudiados se presentan en la Tabla 1.

Tabla 1.- Clasificación y propiedades físico quimicas de los suelos Table 1. Soil classification and thier phisico-chemical properties.

\begin{tabular}{llrrrrrr}
\multicolumn{1}{c}{ Suelo } & Clasificación & C.O. $\%$ & $\mathrm{pH}$ & $\mathrm{Pppm}$ & \multicolumn{3}{c}{$\mathrm{mg} / 100 \mathrm{~g}$ de suelo } \\
& & & & & $\mathrm{K}$ & $\mathrm{Ca}$ & $\mathrm{Mg}$ \\
\hline Colonia Popular & Argiudol típico & 2,0 & 7,0 & 12,0 & 4,0 & 88,0 & 34,0 \\
Tirol & Argiudol típico & 2,0 & 6,0 & 18,0 & 7,0 & 136,0 & 40,0 \\
Mbiguá & Haplustol óxico & 1,0 & 6,0 & 27,0 & 14,0 & 79,0 & 11,0 \\
Los Laureles & Argiudol vértico & 1,0 & 6,5 & 4,0 & 4,0 & 441,0 & 6,0
\end{tabular}

Los métodos utilizados para el análisis de los suelos fueron: para materia orgánica: Walkley y Black modificado; K: fotometría de llama; $\mathrm{P}$ : Bray - Kurtz № 1; Ca y Mg: complejometría EDTA; pH potenciométrico, relación suelo agua 1:2,5. Al final de la experiencia se analizó el suelo de cada maceta con estos mismos métodos. Las muestras se secaron al aire, los terrones se desagregaron manualmente, luego se tamizaron con tamiz de 4 $\mathrm{mm}$.

La especie utilizada fue trébol blanco cv. Haifa (Trifolium repens). Para el ensayo se usaron macetas plásticas con drenaje, las que se llenaron de tierra hasta completar $475 \mathrm{~g}$. Las macetas se colocaron dentro de platos plásticos.

La cantidad de agua necesaria para el riego se calculó según la metodologia propuesta por Chaminade et al (1965). Se regó diariamente agregando el agua en el plato de donde ascendia por capilaridad. De esta manera se trató de evitar una excesiva compactación del suelo.

Se utilizó el concepto de cultivo intensivo en macetas propuesto por Chaminade et al (1965). Se sembraron 250 semillas de trébol blanco por maceta previamente tratadas con frio a $6^{\circ} \mathrm{C}$ durante siete dias e inoculadas con rizobio específico, luego se las cubrió con $25 \mathrm{~g}$ de tierra. Los nutrientes se agregaron como drogas puras: 
$\mathrm{Na} 2 \mathrm{PO} 4 \mathrm{H} .2 \mathrm{H} 2 \mathrm{O} ; \mathrm{KCl} ; \mathrm{CaCO} 3 ; \mathrm{MgCO} 3 ; \mathrm{Na} 2 \mathrm{SO} 4$; $\mathrm{Na2}$ (MoO4); $\mathrm{CuCl} 2$; $\mathrm{ZnCl} 2$ y H3BO3. Las fuentes de $\mathrm{P}, \mathrm{K}, \mathrm{S}, \mathrm{Mo}, \mathrm{Cu}, \mathrm{Zn}$ y $\mathrm{B}$ se agregaron en solución, las de $\mathrm{Ca}$ y $\mathrm{Mg}$ en forma sólida. En los tratamientos que contenían $\mathrm{Ca}$ y $\mathrm{Mg}$ estos se agregaron en primer término, mezclándolos con el suelo, luego se adicionaron las soluciones, volviendo a mezclar.

En los suelos de PuertoTirol y Los Laureles se establecieron diez tratamientos, siguiendo una técnica aditiva: Testigo; P; PK; PKCa; PKCaMg; PKCaMgS; PKCaMgSMo; PKCaMgSMoCu; PKCaMgSMoCuZn; PKCaMgSMoCuZnB. En tanto que en los suelos de Colonia Popular y $\mathrm{A}^{\circ}$ Mbiguá los tratamientos fueron seis, porque no se incluyeron los que contenían micronutrientes. Las dosis de nutrientes agregadas fueron: $P=50 \mathrm{ppm}$; $\mathrm{K}=50 \mathrm{ppm} ; \mathrm{Ca}=500 \mathrm{ppm} ; \mathrm{Mg}=250 \mathrm{ppm} ; \mathrm{S}=5$ $\mathrm{ppm} ; \mathrm{Mo}=5 \mathrm{ppm} ; \mathrm{Cu}=5 \mathrm{ppm} ; \mathrm{Zn}=5 \mathrm{ppm} ; \mathrm{B}=5$ ppm.

Las cosechas se realizaron cortando las plantas a $2 \mathrm{~cm}$ del nivel del suelo con tijeras. La primera cosecha se realizó a sesenta días de la siembra, las siguientes a intervalos de 22 dias, para la segunda y tercera cosecha respectivamente. Se consideró a las deficiencias que aparecian en la primer cosecha como deficiencias primarias, en tanto que las observadas en las cosechas posteriores conformaron deficiencias secundarias (Tomei et al, 1995). El material cosechado se secó en estufa de tiro forzado a $60^{\circ} \mathrm{C}$ hasta peso constante, expresándose los resultados en dg de materia seca (MS).

Se utilizó un diseño estadístico completamente aleatorizado con tres repeticiones. Se realizó el análisis de la variancia, probándose las diferencias entre medias con la prueba de Tukey al nivel $5 \%$.

\section{Resultados y discusión.}

Suelo Colonia Popular: Los resultados obtenidos en cada cosecha y en el total de cosechas en este suelo Colonia Popular (Tabla 2) indican que no hay respuesta significativa al agregado de $P$ y $\mathrm{PK}$, aunque el contenido en el suelo de estos dos nutrientes es relativamente bajo, Tabla 1.

Tabla 2. Producción de materia seca, dg por maceta, en suelo Colonia Popular (Chaco).

Table 2. Dry matter production, dg per pot, in Colonia Popular (Chaco) soil.

\begin{tabular}{lrrr}
\hline Tratamientos & \multicolumn{3}{c}{ Cosechas } \\
& \multicolumn{1}{c}{ Total } \\
\hline Testigo & $8,1 \mathrm{~d}$ & 2 & $14,6 \mathrm{c}$ \\
P & $8,0 \mathrm{~d}$ & $6,5 \mathrm{c}$ & $15,6 \mathrm{c}$ \\
PK & $7,5 \mathrm{~d}$ & $7,6 \mathrm{bc}$ & $13,7 \mathrm{c}$ \\
PKCa & $17,7 \mathrm{a}$ & $6,2 \mathrm{c}$ & $27,7 \mathrm{ab}$ \\
PKCaMg & $15,6 \mathrm{~b}$ & $10,0 \mathrm{bc}$ & $31,7 \mathrm{a}$ \\
PKCaMgS & $13,4 \mathrm{c}$ & $16,0 \mathrm{a}$ & $24,9 \mathrm{~b}$ \\
\hline D.L.S. Tukey $5 \%{ }^{*}$ & 2,1 & $11,5 \mathrm{ab}$ & 4,8 \\
C. V. \%** & 3,8 & 4,9 & 4,7
\end{tabular}

Ref.: Los valores de una misma columna seguidos por letras iguales no difieren entre si $(P>0,05)$. * Diferencia Significativa Limite; ${ }^{*}$ Coeficiente de Variabilidad en \%.

Figures follow by the same letter do not differ between them $(P>0,05)$. "Difference Limit Significative; "* Variability Coefficient $\%$.

En el primer corte los rendimientos logrados con el agregado de $\mathrm{Ca}$, son significativamente diferentes con respecto a los promedios de los tratamientos: testigo, con P y PK, y muestran que constituye una deficiencia primaria. El contenido extremadamente bajo de Ca edáfico explica esta respuesta y limita el efecto del agregado de $P$ solamente. Esto puede explicar los fracasos en las siembras de leguminosas invernales sobre estos suelos.
En el segundo corte se manifiesta el Mg como deficiencia secundaria superando el rendimiento del tratamiento que contiene este nutriente a todos los demás excepto al que posee $S$. Los resultados de la suma de los cortes muestran también que los nutrientes deficientes son $\mathrm{Ca}$ y $\mathrm{Mg}$. Considerando que el $\mathrm{pH}$ original del suelo está dentro de limites aceptables para el $T$. repens los resultados logrados con el agregado de estos elementos podrían indicar que se deben a su rol 
como nutrientes. Sin embargo se produce un cambio significativo en los valores de $\mathrm{pH}$ con el agregado de carbonato de calcio (Tabla 3 ). También se determina un aumento estadísticamente significativo en $\mathrm{P}$ disponible en el suelo. Si bien existe un efecto favorable sobre el crecimiento del trébol con el agregado de $\mathrm{Ca}$ y $\mathrm{Mg}$, no queda claro si se debe solo a sus funciones como nutrientes o si se trata de un efecto más complejo que puede involucrar a la mayor disponibilidad de $\mathrm{P}$ en la solución del suelo.

Tabla 3. Suelo Colonia Popular, análisis final de suelos.

Table 3. Colonia Popular soil, final soil analysis

\begin{tabular}{|c|c|c|c|c|c|}
\hline \multirow[t]{2}{*}{ Tratamientos } & \multirow[b]{2}{*}{$\mathrm{pH}$} & \multirow[b]{2}{*}{ P ppm } & \multicolumn{3}{|c|}{$\mathrm{mg} / 100 \mathrm{~g}$} \\
\hline & & & $\mathrm{K}$ & $\mathrm{Ca}$ & $\mathrm{Mg}$ \\
\hline Testigo & $6,7 \mathrm{~b}$ & $7,2 d$ & $4,4 d$ & $114,3 b$ & $41,3 b$ \\
\hline$P$ & $6,7 b$ & $31,5 b$ & $5,2 b$ & $118,2 b$ & $26,8 b$ \\
\hline PK & $6,7 \mathrm{~b}$ & $27,2 \mathrm{c}$ & $5,7 a$ & $118,2 b$ & $59,2 \mathrm{a}$ \\
\hline $\mathrm{PKCa}$ & $8,3 a$ & $36,3 a$ & $5,1 b$ & $176,4 a$ & $31,2 b$ \\
\hline PKCaMg & $8,3 a$ & $37,9 a$ & $5,1 \mathrm{~b}$ & $208,4 a$ & $65,8 \mathrm{a}$ \\
\hline PKCaMgS & $8,3 a$ & $33,6 \mathrm{~b}$ & $4,8 \mathrm{c}$ & $214,4 a$ & $65,8 \mathrm{a}$ \\
\hline D.L.S. Tukey $5 \%$ & 0,2 & 2,4 & 0,3 & 46,1 & 16,7 \\
\hline C. V. $\%$ & 0,7 & 1,8 & 1,3 & 6,1 & 7,4 \\
\hline
\end{tabular}

Ref.: Idem Tabla 2.

Suelo Puerto Tirol: En este suelo se hicieron tres cosechas (Tabla 4), en la primera solo hubo un efecto positivo no significativo con el agregado de P. El agregado de los otros nutrientes provocó una disminución en los rendimientos dando diferencias significativas entre las medias. Estos resultados indican que no existen deficiencias nutricionales minerales primarias. Hubo un efecto depresivo por exceso del conjunto de cationes agregados.
En la segunda cosecha existe respuesta significativa al agregado de $\mathrm{Ca}$ indicando que constituye una deficiencia secundaria. Es probable que en los tratamientos sin este nutriente el cultivo intensivo agote rápidamente el $\mathrm{Ca}$ disponible, siendo su tasa de reposición muy lenta a partir de las fracciones lábiles. O bien la entidad de estas fracciones es muy baja, predominando las más estables.

Tabla 4. Producción de materia seca, dg por maceta, en suelo de PuertoTirol (Chaco).

Table 4. Dry matter production, dg per pot, in Puerto Tirol (Chaco) soil.

\begin{tabular}{|c|c|c|c|c|}
\hline \multirow[t]{2}{*}{ Tratamientos } & \multicolumn{4}{|c|}{ Cosechas } \\
\hline & 1 & 2 & 3 & Total \\
\hline Testigo & $16,8 a b$ & $5,8 c$ & $4,4 \mathrm{~b}$ & $27,0 \mathrm{c}$ \\
\hline$P$ & $17,7 a$ & $5,8 \mathrm{c}$ & $5,9 \mathrm{~b}$ & $29,4 \mathrm{C}$ \\
\hline PK & $14,8 a b c$ & $6,7 c$ & $5,2 b$ & $26,7 \mathrm{C}$ \\
\hline $\mathrm{PKCa}$ & $15,4 a b c$ & $11,6 a$ & $17,2 \mathrm{a}$ & $44,2 a$ \\
\hline PKCaMg & $13,3 \mathrm{c}$ & $11,8 \mathrm{a}$ & $18,7 a$ & $43,9 a b$ \\
\hline PKCaMgS & $12,8 \mathrm{c}$ & $11,2 \mathrm{a}$ & $19,3 a$ & $43,3 a b$ \\
\hline PKCaMgSMo & $12,7 \mathrm{C}$ & $11,4 a$ & $19,5 a$ & $43,6 a b$ \\
\hline PKCaMgSMoCu & $13,3 \mathrm{c}$ & $10,9 a$ & $16,0 a$ & $40,2 a b$ \\
\hline PKCaMgSMoCuZn & $13,9 b c$ & $11,5 a$ & $16,0 \mathrm{a}$ & $41,0 a b$ \\
\hline PKCaMgSMoCuZnB & $9,3 d$ & $11,0 \mathrm{a}$ & $19,5 a$ & $39,8 \mathrm{~b}$ \\
\hline D.L.S. Tukey $5 \%$ & 3,2 & 1,3 & 3,7 & 4,3 \\
\hline C. V. $\%$ & $4, \overline{6}$ & 2,7 & 5,1 & 2,2 \\
\hline
\end{tabular}

Ref.: Idem Tabła 2. 
Estos resultados se repiten en la tercer cosecha y en la suma de las tres realizadas. En la tercer cosecha la acción favorable del $\mathrm{Ca}$ sobre los rendimientos observada en la segunda es más evidente, mientras que los tratamientos que incluyen $\mathrm{Mg}$ y $\mathrm{S}$ los mejoran en forma no significativa.

Suelo $A^{\circ}$ Mbiguá: Con este suelo se hicieron dos cosechas, no se registran diferencias significativas entre tratamientos (Tabla 5), indicando que no se manifiestan deficiencias de los nutrientes estudiados. Este suelo esta ubicado en el albardón, de pendiente suave del Arroyo Mbiguá, los que son utilizados en agricultura. El análisis químico muestra un contenido mediano en $P$ hecho que justifica la falta de respuesta al agregado de este nutriente. En este suelo no se estudió el efecto del agregado de micronutrientes.

Tabla 5. Producción de materia seca, dg por maceta, en suelo de $A^{\circ}$ Mbiguá (Formosa).

Table 5. Production of dry matter, dg per pot, in $A^{0}$ Mbiguá (Formosa) soil.

\begin{tabular}{lrrr} 
Tratamientos & \multicolumn{3}{c}{ Cosechas } \\
& 1 & 2 & Total \\
\hline Testigo & $13,9 a$ & $6,2 a$ & $20,0 a$ \\
P & $15,9 a$ & $6,7 a$ & $22,6 a$ \\
PK & $17,2 a$ & $6,3 a$ & $23,5 a$ \\
PKCa & $17,2 a$ & $6,1 a$ & $23,3 a$ \\
PKCaMg & $14,3 a$ & $6,0 a$ & $20,3 a$ \\
PKCaMgS & $16,7 a$ & $6,1 a$ & $22,7 a$ \\
\hline D.L.S. Tukey 5\% & 3,4 & 1,9 & 4,6 \\
\hline C. V. \% & 4,8 & 6,8 & 4,6
\end{tabular}

Ref.: Idem Tabla 2.

Suelo Los Laureles: Dio tres cosechas, en la primera se manifiestan deficiencias primarias de $P$ y $\mathrm{Zn}$ (Tabla 6), la deficiencia primaria en este último micronutriente es un hecho no observado aún en este tipo de ensayos con suelos del Noreste Argentino. Estas deficiencias explicarian los fracasos en la implantación de leguminosas invernales en estos suelos. Por otra parte Mufarrege (1995) hace referencia a contenidos de $\mathrm{Zn}$ en pasturas naturales del área en estudio que están por debajo de los niveles críticos para los bovinos. La deficiencia en $\mathrm{Zn}$ en el suelo puede

Tabla 6. Producción de materia seca, dg por maceta, en suelo Los Laureles (Santa Fe). Table 6. Dry matter production, dg per pot, in Los Laureles (Santa Fe) soil.

\begin{tabular}{lrrrr}
\hline Tratamientos & \multicolumn{4}{c}{ Cosechas } \\
& 1 & 2 & 3 & Total \\
Testigo & $3,5 \mathrm{c}$ & $3,7 \mathrm{e}$ & $4,0 \mathrm{~d}$ & $11,2 \mathrm{f}$ \\
P & $10,8 \mathrm{~b}$ & $6,5 \mathrm{~d}$ & $5,8 \mathrm{~cd}$ & $21,7 \mathrm{e}$ \\
PK & $9,0 \mathrm{~b}$ & $7,0 \mathrm{~cd}$ & $5,8 \mathrm{~cd}$ & $23,1 \mathrm{de}$ \\
PKCa & $9,9 \mathrm{~b}$ & $7,8 \mathrm{c}$ & $6,7 \mathrm{bc}$ & $24,4 \mathrm{de}$ \\
PKCaMg & $10,2 \mathrm{~b}$ & $8,2 \mathrm{c}$ & $7,9 \mathrm{~b}$ & $26,3 \mathrm{~cd}$ \\
PKCaMgS & $11,7 \mathrm{~b}$ & $10,9 \mathrm{a}$ & $10,3 \mathrm{a}$ & $32,9 \mathrm{~b}$ \\
PKCaMgSMo & $12,0 \mathrm{~b}$ & $9,6 \mathrm{~b}$ & $10,9 \mathrm{a}$ & $32,5 \mathrm{~b}$ \\
PKCaMgSMoCu & $13,0 \mathrm{~b}$ & $9,6 \mathrm{~b}$ & $8,0 \mathrm{~b}$ & $30,6 \mathrm{bc}$ \\
PKCaMgSMoCuZn & $18,4 \mathrm{a}$ & $11,1 \mathrm{a}$ & $9,8 \mathrm{ab}$ & $39,2 \mathrm{a}$ \\
PKCaMgSMoCuZnB & $13,2 \mathrm{~b}$ & $7,7 \mathrm{c}$ & $10,8 \mathrm{a}$ & $31,7 \mathrm{~b}$ \\
\hline D. L. S. Tukey $5 \%$ & 4,8 & 1,2 & $-\frac{1}{2}, 1$ & 4,5 \\
\hline C. V. \% & 8,5 & 4,6 & 7,6 & 4,9 \\
\hline
\end{tabular}

Ref.: Idem Tabla 2. 
ser la causa de la baja respuesta de los tréboles que se observa en el campo cuando solo se fertiliza con superfosfato triple.

Las deficiencias en $P$, detectadas por métodos biológicos, son frecuentes en suelos de la Provincia de Corrientes (Tomei et al, 1995), aunque no se han mencionado para suelos chaqueños.

A pesar del bajo contenido en $\mathrm{K}$ de este suelo, el agregado de este nutriente no produce respuesta en ninguna de las cosechas realizadas.

En la segunda cosecha el Ca y el $S$ se manifiestan como deficiencias secundarias, estos nutrientes pueden ser limitantes del crecimiento de tréboles a largo plazo. Estos resultados se repiten en la tercera cosecha, no manifestándose otros nutrientes deficientes. El suelo está bien provisto de $\mathrm{Ca}$ de manera que la respuesta observada al agregado de este nutriente probablemente se deba a restricciones en disponibilidad (Killian y Velly, 1964). El $S$ disponible para las plantas proviene de la mineralización de la materia orgánica (MO) del suelo. En este caso la velocidad del proceso de mineralización y el contenido bajo en $\mathrm{MO}$, es posible que determinen la deficiencia secundaria en $\mathrm{S}$.

De los cuatro suelos estudiados solo en uno de ellos se observa deficiencia primaria de $P$, en el mismo suelo, (Los Laureles, Santa Fe) se detecta deficiencia primaria de Zn. En un suelo, Colonia Popular (Chaco), se determina una deficiencia primaria de $\mathrm{Ca}$. Tres de los suelos tienen deficiencias secundarias de $\mathrm{Mg}$ y en uno de ellos (Puerto Tirol, Chaco) también lo es el Ca. Solo el suelo Mbiguá, Formosa, no presenta limitaciones de nutrientes minerales para el trébol blanco. Los resultados indican que los diferentes suelos del Chaco Oriental pueden presentar restricciones de nutrientes minerales para el establecimiento y persistencia de leguminosas forrajeras invernales.

\section{Bibliografía.}

- Chaminade, R. 1965. Bilan de trois années d'experimentation en petits vases de végétation. L'Agronomie Tropicale 20(11): 1101-1162.

- Chaminade, R. 1968. Theories scientifiques de la fertilizacion des sols. II. Compte rendus des debats. B.Diagnistic des carences. L'Agronomie Tropicale 23 (2): 185-186.

- Espino, L.M. y M.A. Seveso. 1983. Mapas de suelos de la Provincia de Santa Fe. INTA - MAG. 216 p.

- Killian, J. y J. Velly 1964. Diagnostic des carences minerales en vases de vegétation sur quelques sols de Madagascar. L'Agronomie Tropicale 19(5):413 - 443.

- Ledesma, L.L. 1990. Atlas de Suelos de la República Argentina. Suelos del Chaco. SAG y P - INTA - PNUD Arg/85/019. Vol. l:250 - 331

- Mufarrege, D.J. 1995. La práctica de la suplementación mineral del ganado en la región NEA. Ganaderia Subtropical 95, Jornadas Internacionales de Actualización. pp $41-50$.

- Roche, P y J. Velly. 1965. Les métodes utilisées pour reconstituer la fertilite des sols tropicaux. Etudes de carences. Essais aux champs. Curbe de réponse. Resultats obtenus a Madagascar. L'Agronomie Tropicale 20(10): 1029-1033.

- Roche, P. 1967. Contribution a l'étude du statut phosphorique des sols de Madagascar. Incidence sur les probiemes de fertilité. L'Agronomie Tropicale 22(3): 249 308.

- Schenkel, G. 1971. Evaluación de la fertilidad de un suelo. mediante la producción de materia seca en ensayos en macetas. I. Representaciones gráficas usadas. Turrialba Vol. 21 (3): 253-262.

- Tomei, C.E. y P.M. Fuentes Godo. 1974. Contribucion al conocimiento de las relaciones entre el agregado de fertilizantes y la producción de leguminosas en suelos de la Provincia de Corrientes. II Rol de diferentes nutrientes en la productividad primaria. II Reunión Nacional de Fertilidad y Fertilizantes. Sociedad Científica Argentina. Buenos Aires. p. $254-264$.

- Tomei. C.E., M.E. Castelan, M.M. Poletti y M.A. Slukwa. 1995. Respuesta del Trifolium repens L. al agregado de $P, K, C a, M g$ y $S$ en ocho suelos del Nordeste Argentino. Revista de la Facultad de Agronomia. Facultad de Ciencias Agrarias y Forestales. UNLP. Tomo 71 (2): $173-178$. 